\title{
Neuronal jitter: can we measure the spike timing dispersion differently? Lubomir Kostal ${ }^{* 1}$ and Petr Marsalek 2,3
}

\author{
Address: ${ }^{1}$ Institute of Physiology, Czech Academy of Sciences, Videnska 1083, Praha 4, 142 20, Czech Republic, ${ }^{2}$ First Medical Faculty, Charles \\ University of Prague, U nemocnice 5, 128 53, Praha, Czech Republic and ${ }^{3}$ Czech Technical University, Faculty of Biomedical Engineering, Nam. \\ Sitna 3105, 27201 Kladno, Czech Republic \\ Email: Lubomir Kostal* - kostal@biomed.cas.cz \\ * Corresponding author
}

from Eighteenth Annual Computational Neuroscience Meeting: CNS*2009

Berlin, Germany. 18-23 July 2009

Published: 13 July 2009

BMC Neuroscience 2009, I0(SuppI I):PI03 doi:I0.II86/I47I-2202-I0-SI-PI03

This abstract is available from: http://www.biomedcentral.com/I47I-2202/I0/SI/PI03

(c) 2009 Kostal and Marsalek; licensee BioMed Central Ltd.

To measure the spike timing precision is a tedious task. First, spike trains may be interleaved by bursts and interpreting their information content is difficult [1]. Second, input-output neuronal transfer functions are nonlinear, depend on many synaptic inputs and it is also difficult to track the source of variability in experimental data. We propose an information-theoretic quantity, entropybased dispersion (ED), to measure dispersion of a continuous spike-timing probability distribution. The method is based on the notions of differential entropy and asymptotic equipartition property [2]. The ED is conceptually similar to the traditionally used standard deviation (SD) and coefficient of variation (CV). However, we show that the properties of SD and ED are different: while SD is a second-moment characteristics measuring the dispersion relative to the mean value, $\mathrm{ED}$ measures the absolute effective spread of the probability distribution and is more closely related to the concept of randomness [3].

We apply both measures to analyze the temporal precision of neuronal spiking activity of the perfect integrateand-fire model [4], which is a plausible neural model if the synaptic activity is high enough. The synaptic activity is described by several frequently used models, namely, the inter-spike interval (ISI) probability distribution of presynaptic neurons is either exponential, gamma, inverse Gaussian or bimodal lognormal.
We show that SD and ED behave similarly for simple models of presynaptic activity (Poisson, gamma and inverse Gaussian) when the CV of input spike trains interspike intervals is not greater than 1 . However, for bimodal lognormal or overdispersed input spike trains $(\mathrm{CV}>1)$ the results obtained by SD and ED differ strikingly. We discuss these differences and argue, that contrary to ED, SD may overestimate or underestimate the variability of output spiking activity. We show on several examples, how the ED might pinpoint some optimal regimes or parameter values of spike trains.

\section{Acknowledgements}

This work was supported by the research projects MSM 0021620806 and MSM 6840770012 granted by the Ministry of Education, Youth and Sports of the Czech Republic, by research project AVOZ50I 10509, Centre for Neuroscience LC554 and by the Academy of Sciences of the Czech Republic grant KJBI00I I070I.

\section{References}

I. Bair W, Koch C, Newsome W, Britten K: Power spectrum analysis of bursting cells in area MT in the behaving monkey. J Neurosci 1994, 14:2870-2892.

2. Cover TM, Thomas JA: Elements of Information Theory New York: John Wiley \& Sons; 1991.

3. Kostal L, Lansky P, Rospars JP: Neuronal coding and spiking randomness. Eur J Neurosci 2007, 26:2693-270I.

4. Marsalek P, Koch C, Maunsell J: On the relationship between synaptic input and spike output jitter in individual neurons. Proc Natl Acad Sci USA 1997, 94:735-740. 